\title{
Effect of CYP3A5 polymorphism on liver function and tacrolimus pharmacokinetics after conversion to a once-daily tacrolimus formulation in stable liver transplant patients
}

\author{
Jong Man Kim ${ }^{1}$, Je Ho Ryu ${ }^{2}$, Kwang-Woong Lee ${ }^{3}$, Suk Kyun Hong ${ }^{3}$, Kwangho Yang ${ }^{2}$, \\ Gyu-Seong Choi ${ }^{1}$, Young-ae Kim ${ }^{4}$, Ju-Yeun Lee ${ }^{5}$, Nam-Joon $\mathrm{Yi}^{3}$, Choon Hyuck Kwon ${ }^{6}$, \\ Chong Woo Chu ${ }^{7}$, Kyung-Suk Suh ${ }^{3}$, and Jae-Won Joh ${ }^{1}$ \\ ${ }^{1}$ Samsung Medical Center \\ ${ }^{2}$ Pusan National University Yangsan Hospital \\ ${ }^{3}$ Seoul National University College of Medicine \\ ${ }^{4}$ Seoul National University Hospital \\ ${ }^{5}$ Seoul National University \\ ${ }^{6}$ Cleveland Clinic \\ ${ }^{7}$ Good Gangan Hospital
}

June 18, 2020

\begin{abstract}
Aim: To analyze the effects of CYP3A5 polymorphism on liver function after LT and to characterize the pharmacokinetics of tacrolimus after conversion from a twice-daily regimen to a once-daily extended-release formulation. Methods: A prospective open-label study included 60 stable liver transplant recipients who underwent 1:1 conversion from twice-daily tacrolimus to oncedaily tacrolimus. All participants were genotyped for CYP3A5 polymorphism. The study was registered at ClinicalTrials.gov (NCT 02882113). Results: Twenty-eight patients were enrolled in the CYP3A5 expressor group and 32 in the non-expressor group. Although there was no statistical difference, incidence of liver dysfunction was higher in the expressor group than in the non-expressor group when converted to once-daily extended-release tacrolimus $(\mathrm{P}=0.088)$. No biopsy-proven acute rejection, graft failure, and mortality were observed in either group. The decrease in dose-adjusted trough level (- 42.9\% vs. - 26.1\%) and dose/kg-adjusted trough level of tacrolimus (- $40.0 \%$ vs. - 23.7\%) was significantly greater in the expressor group than in the non-expressors after the conversion. The absorption of the tacrolimus in the non-expressor group was slower than in the expressors. In line with this observation, the AUC for once-daily tacrolimus correlated with Cmin in the non-expressors and Cmax in the expressors. Conclusions: Determination of CYP3A5 genotype in liver transplant recipients might be helpful in prediction of tacrolimus pharmacokinetics after conversion from a twice-daily regimen to a once-daily formulation.
\end{abstract}

\section{INTRODUCTION}

Tacrolimus is an effective immunosuppressant, and its use in liver transplantation (LT) is well established. ${ }^{1}$ However, the drug has a narrow therapeutic window, and its pharmacokinetics and pharmacodynamics vary considerably at both intraindividual and interindividual level; hence, establishing an empirical dosage regimen may pose a challenge..$^{2-4}$

As a result of recent advances in pharmacogenomics, several single nucleotide polymorphisms in the intron 3 of cytochrome P450 (CYP )3A5 were identified and were shown to correlate with the expression of the gene and enzymatic activity of the product it encodes. ${ }^{4}$ The intra- and interindividual variability in tacrolimus pharmacokinetics and pharmacodynamics is partly associated with the polymorphism of the 
genes for cytochrome $\mathrm{P} 450$ enzymes, CYP3A4 and CYP3A5 , and the efflux transporter P-glycoprotein (Pgp). ${ }^{4}$ Polymorphism at a cryptic splice site may result in either the presence (expressor, ${ }^{*} 1 /{ }^{*} 1$ and ${ }^{*} 1 /{ }^{*} 3$ ) or absence (non-expressor, ${ }^{*} 3{ }^{*} 3$ ) of CYP $3 A 5 .{ }^{4}$ The frequencies of CYP3A5 polymorphism differ depending on race, ${ }^{4,5}$ with the CYP3A5 expressors (i.e., ${ }^{*} 1{ }^{*} 1$ or ${ }^{*} 1 /{ }^{*} 3$ ) and MDR1 C3435Twild-type C allele carriers (i.e., $C C$ or $C T$ ) being more prevalent among Asians (51\% and $62.1 \%$, respectively) than in Caucasians (10\% and $43.4 \%$, respectively). ${ }^{6}$

Several studies demonstrated that the expression of $C Y P 3 A 5$ results in a lower tacrolimus exposure, and hence, CYP3A5expressors might require a higher dose of the drug than non-expressors. ${ }^{2,3,7,8}$ While we still know little about the effect of $C Y P 3 A 5$ genotype on patient performance post-transplant, it has been shown to affect tacrolimus pharmacokinetics. A once-daily extended-release formulation (Advagraf ${ }^{\circledR}$ ) has been approved in many countries since 2007. Several studies have demonstrated that in stable patients after LT, conversion from twice-daily to once-daily tacrolimus was well-tolerated, safe and convenient. ${ }^{9,10}$ However, despite similar pharmacokinetics of the twice-daily and once-daily tacrolimus, some patients experienced adverse events, including liver dysfunction, after the conversion. ${ }^{11}$

To the best of our knowledge, none of the previous studies analyzed a link between CYP3A5 polymorphism and the changes in tacrolimus pharmacokinetics after conversion from the twice-daily regimen to a once-daily formulation. Therefore, the aim of this study was to compare the incidence of liver dysfunction in stable liver transplant recipients, both CYP3A5 expressors and non-expressors, after conversion to once-daily expandedrelease tacrolimus (Advagraf ${ }^{\circledR}$ ), and to analyze the effect of CYP3A5 genotype on the pharmacokinetics of both a twice-daily regimen and a once-daily tacrolimus formulation.

\section{MATERIALS AND METHODS}

\section{Study design}

This prospective, multicenter, open-label, exposure-variable study was carried out at the Samsung Medical Center (Seoul, Korea), Seoul National University Hospital (Seoul, Korea), and Yangsan Pusan National University Hospital (Yangsan, Busan) between August 2016 and June 2018. The study was carried out in accordance with the Declaration of Helsinki and was registered at ClinicalTrials.gov (NCT 02882113). The protocol of the study was approved by the Institutional Review Boards at the Samsung Medical Center (IRB No. 2015-11-140), Seoul National University Hospital (IRB No. H-1510-097-712), and Yangsan Pusan National University Hospital (IRB No. 04-2015-038). All participants provided written informed consent to participate in the study.

\section{Patients}

The study participants received LT more than six months but no earlier than three years before the enrollment. The inclusion criteria were: age [?]19 years, the use of twice-daily tacrolimus at screening, stable kidney function (serum creatinine level, $\mathrm{Cr}<2.0 \mathrm{mg} / \mathrm{dL}$ ) and liver function (serum aspartate aminotransferase (AST) and alanine aminotransferase (ALT) levels within normal ranges), and maintenance of the same immunosuppressive dosing regimen for [?]2 weeks before the enrollment. The patients who received any drugs known to interfere with tacrolimus pharmacokinetics and those enrolled in other immunosuppressant study protocols were not eligible for the study. Other exclusion criteria were: trough level of tacrolimus at screening $<2 \mathrm{ng} / \mathrm{mL}$, an acute rejection episode within 90 days before the enrollment, other organ transplant, renal dysfunction (serum $\mathrm{Cr}$ [?]2 $\mathrm{mg} / \mathrm{dL}$ or estimated glomerular filtration rate, eGFR $<30 \mathrm{~mL} / \mathrm{min}$ ), clinically significant infection, a history of malignancy other than hepatocellular carcinoma or skin cancer, recurrent hepatitis B virus, or hepatitis $\mathrm{C}$ virus infection, liver dysfunction, pregnancy, or unstable concurrent medical condition.

This was not a hypothesis driven study, but a pilot study to investigate the effect of CYP3A5 polymorphism on liver function and trough level of tacrolimus after conversion to the once-daily extended-release tacrolimus formulation. The study patients were grouped based on the expressions of CYP3A5 polymorphism (expressors vs. non-expressors). The minimum required sample size was 30 patients per group. This number was 
based on a general assumption that the variance for a sample of at least 30 reflects the population variance quite accurately, and hence, statistical testing that does not target a specific hypothesis is valid. Therefore, our goal was to recruit a total of 60 patients, 30 per group. The dropout rate was not considered in the safety-oriented analysis. In addition, we performed a pharmacokinetic analysis in 10 patients (5 per group) who agreed to participate in this part of the study.

\section{Tacrolimus concentrations}

Once-daily tacrolimus was administered at 8 A.M., and the dose was adjusted according to the daily trough level of the drug ( $\mathrm{C} 0$ or $\mathrm{Cmin}$ ). The trough levels of tacrolimus were measured by liquid chromatographytandem mass spectrometry using a Waters 2795 Alliance HT system (Waters Ltd., Watford, UK) and a Quattro micro API tandem mass spectrometer (Micromass, Manchester, UK). Before and after the conversion, all tacrolimus doses were adjusted according to the trough level of the drug, to obtain a therapeutic window of $2-8 \mathrm{ng} / \mathrm{mL}$.

\section{Genotyping of cytochrome P450 345}

Genomic DNA was extracted from whole blood samples using a QIAamp DNA Mini kit (Qiagen Inc., Germantown, MD, USA), and stored at - 20degC. The genotypes of the CYP3A5 gene (6986 A>G in intron 3 ) and the $A B C B 1$ gene (1236C $>T$ in exon 12 and $3435 C>T$ in exon 26) were determined using the TaqMan allelic discrimination assay. Primers and probes were designed with Primer Express Software Version 3.0 (Applied Biosystems, Foster City, CA, USA). The polymerase chain reaction (PCR) mixture included $50 \mathrm{ng}$ of genomic DNA in a $1 \mu \mathrm{L}$ volume, along with the following reagents: FAM and TET probes $(5 \mathrm{pmol} / \mu \mathrm{L}$, respectively), primers $(20 \mathrm{pmol} / \mu \mathrm{L}$ for sense and antisense primers, respectively), and $2 \mathrm{X}$ TaqMan Universal PCR Master Mix (Applied Biosystems). PCR cycling reactions were conducted in an ABI 9700 PCR system (Applied Biosystems); the reactions consisted of initial 10-min denaturation at $95^{\circ} \mathrm{C}$, followed by 40 cycles of 15 -sec denaturation at $95^{\circ} \mathrm{C}$ and 1 -min annealing and extension at $60^{\circ} \mathrm{C}$. The results were analyzed with the ABI 7900HT Sequence Detection System (Applied Biosystems). To control the genotyping quality, 10\% of the samples were randomly selected for retyping with a double-blind check. Genetic polymorphisms were analyzed on the day of transplantation. The study patients were blinded to the genetic polymorphisms they carried.

Considering the low frequency of $C Y P 3 A 5^{*} 1$ allele, its carriers $\left(C Y P 3 A 5^{*} 1{ }^{*} 1\right.$ or $\left.C Y P 3 A 5^{*} 1{ }^{*} 3\right)$ were selected first and classified as the "expressors". The patients who carried $C Y P 3 A 5^{*} 3 /{ }^{*} 3$ genotype, responsible for the lack of $C Y P 3 A 5$ expression, were selected second, and were classified as the "non-expressors".

\section{Immunosuppression}

Immunosuppressive therapy after LT was based on the combination of calcineurin inhibitor (tacrolimus) and mycophenolate mofetil (MMF). Two types of twice-daily tacrolimus were used before the conversion: the reference tacrolimus product (Prograf ${ }^{\circledR}$; Astellas Pharma, Tokyo, Japan) and the generic formulation of tacrolimus (Tacrobell ${ }^{\circledR}$; Chong Kun Dang Pharma, Seoul, Korea). All patients were converted to oncedaily tacrolimus (Advagraf ${ }^{\circledR}$, Astellas Pharma, Inc., Deerfield, IL, USA) on a 1:1 mg basis for the total daily dose. However, some dose adjustments were permitted depending on the patient's condition, and other immunosuppressants were allowed to be used according to standard practice. The serum trough levels of tacrolimus and clinical assessments for safety and rejection were completed four weeks after the conversion; then, parameters were evaluated routinely according to the patient's follow-up schedule. The doses of tacrolimus at baseline and during follow-up were adjusted on an individual basis according to the serum trough level of the drug.

\section{Endpoints}

The primary endpoint of this study was the incidence of liver dysfunction that required discontinuation or dosage modification of tacrolimus from registration to six months. The liver dysfunction was defined as an AST or ALT level greater than two times the upper limit of normal. The secondary endpoints were tacrolimus trough level changes and the incidence of acute rejection. The rejection was defined according to the Banff 
criteria for liver biopsy ${ }^{12,13}$. Additionally, the changes in pharmacokinetic parameters of tacrolimus before and after the conversion to once-daily extended-release formulation were analyzed.

\section{Pharmacokinetic Analysis}

The pharmacokinetics of tacrolimus was evaluated within seven days after the enrollment, based on the drug levels in 13 blood samples ( $3 \mathrm{~mL}$ each) collected at 0 (pre-dose) and 0.5, 1, 1.5, 2, 3, 4, 6, 9, 12, 16, 20, and $24 \mathrm{~h}$ after the morning dose. All blood samples were collected to ethylenediaminetetraacetic acid-containing Vacutainer $(\mathrm{R})$ tubes. and were stored at $4^{\circ} \mathrm{C}$ until the analysis was carried out on the same day. The trough levels ( $\mathrm{C} 0$ and $\mathrm{Cmin}$ ) were measured just before tacrolimus administration; peak tacrolimus concentration (Cmax) for each subject was obtained directly from the raw data. The area under the curve (AUC) was estimated using the linear trapezoidal method from hours 0 to 24.

\section{Statistical analysis}

Statistical analyses were conducted using SPSS software (version 22.0, SPSS Inc., Chicago, IL, USA). The data are expressed as medians and ranges or as frequencies (percentages). All tests were two tailed, and $P$ $<0.05$ was considered significant. The values of continuous variables in the expressors and non-expressors were compared using the Mann-Whitney $U$ test, and the Wilcoxon test was used to compare the value of continuous variables within the same group. The distributions of categorical variables were compared with the Fisher exact test. The effect of CYP3A5 polymorphism on changes in tacrolimus exposure after the conversion to once-daily formulation was tested during the analysis of covariance (ANCOVA).

\section{RESULTS}

\section{Patient characteristics}

Eighty-six patients were screened for enrollment to this study. A total of 24 patients were excluded at the screening because of the surplus of CYP3A5 non-expressors (as stated above, the target size of this group was defined as approximately 30). As another two patients were excluded due to overdose of tacrolimus after the conversion to the once-daily extended-release formulation, the study included 60 patients, 28 expressors and 32 non-expressors group among them (Figure 1). The full analysis set (FAS) included all eligible patients who received [?]1 dose of once-daily extended release tacrolimus, with the patients analyzed according to the treatment allocation. The per-protocol (PP) set included all patients who completed the study without a major protocol deviation.

The non-expressor group included a significantly higher proportion of men than the expressor group (77.4\% vs. $42.9 \%, \mathrm{P}=0.008)$. The two study groups did not differ significantly in terms of other clinical characteristics or in the time elapsed from the liver transplantation to the study recruitment (Table 1).

\section{Efficacy}

In the full analysis set (FAS), the incidence of liver dysfunction after the conversion to once-daily extendedrelease tacrolimus was $17.9 \%$ in the expressor group $(\mathrm{n}=5)$ versus $3.1 \%(\mathrm{n}=1)$ in the non-expressor group $(\mathrm{P}=0.088)$. In the per-protocol $(\mathrm{PP})$ set, the incidence of liver dysfunction in the expressor group was higher than in the non-expressor group $(12.0 \%$ vs. $0 \%, \mathrm{P}=0.088)$. The conversion to once-daily extendedrelease tacrolimus was not associated with acute rejection, graft failure, and mortality. The expressor and non-expressor groups did not differ significantly in terms of serum creatinine levels and liver parameters, such as AST, ALT, total bilirubin, and alkaline phosphatase (ALP) during the follow-up (Figure 2).

\section{Tacrolimus dose adjustments after the conversion}

Eight patients from the expressor group (28.6\%) and seven patients from the non-expressor group (21.9\%) required an increase in the dose of extended-release tacrolimus during the stud period. The dose of tacrolimus was decreased in two patients from the expressor group (7.1\%) and in three patients from the non-expressor group (9.4\%). The frequency of tacrolimus dose adjustment did not differ significantly between the study groups $(\mathrm{P}=0.787)$. 


\section{Tacrolimus exposure before and after the conversion}

The main parameters of tacrolimus exposure determined in both study groups (expressors and nonexpressors) determined after administration of the twice-daily and once-daily formulations are summarized in Table 2, and the blood concentration-time profiles of tacrolimus are shown in Figure 3. After the administration of tacrolimus, the median daily dose of the drug and the median dose/weight were significantly higher in the expressor group $(\mathrm{P}<0.001)$. Despite this, the study groups did not differ significantly in terms of the median tacrolimus trough levels during the follow-up. The median dose-adjusted trough level $(\mathrm{C} 0 /$ dose $)$ and dose $/ \mathrm{kg}$-adjusted trough level $(\mathrm{C} 0 / \mathrm{dose} / \mathrm{kg})$ were significantly lower in the expressor group than in the non-expressor group $(\mathrm{P}<0.001)$.

After the conversion to once-daily extended-release tacrolimus, median trough level of the drug $(\mathrm{C} 0)$ decreased by nearly $25 \%$; the change was similar regardless of the CYP3A5 genotype (- $24.0 \%$ in the expressor group vs. $-25.5 \%$ in the non-expressor group). Moreover, a decrease in the median dose-adjusted trough level and dose/kg-adjusted trough level was observed, by $42.9 \%$ and $40.0 \%$ in the expressor group, respectively, and by $26.1 \%$ and $23.7 \%$ in the non-expressor group, respectively. The decrease in the dose-adjusted trough level and dose/kg-adjusted trough level was significantly greater in the expressor group than in the non-expressor group $(\mathrm{P}<0.001$ and $\mathrm{P}<0.001$, respectively).

\section{Pharmacokinetics before and after the conversion}

Pharmacokinetic findings of this study are summarized in Table 3. Mean whole blood concentrations of tacrolimus before and after the conversion are depicted in Figure 3. No statistically significant differences were found in the area under the curve (AUC), Cmin, Cmax, dose-adjusted Cmin, dose-adjusted Cmax, dose-adjusted AUC, dose/kg-adjusted Cmin, dose/kg-adjusted Cmax, and dose/kg-adjusted AUC for the between the twice-daily tacrolimus and once-daily tacrolimus groups. The median dose of tacrolimus was higher in the expressor group than in the non-expressor group, while median dose/weight, Cmax in twicedaily tacrolimus/dose, AUC in twice-daily tacrolimus, and dose/kg-adjusted Cmax in twice-daily tacrolimus in the expressors were significantly lower than in the non-expressors (Table 3). Median AUC values for the twice-daily tacrolimus and once-daily tacrolimus were higher in the expressor group than in the non-expressor group, but the differences were not significant. In the expressor group, the Cmax for once-daily tacrolimus was higher than the Cmax for twice-daily tacrolimus, whereas an inverse relationship was observed in the non-expressor group. In line with these findings, significant correlations were found between the Cmin and AUC for once-daily tacrolimus in the non-expressor group, and between the Cmax and AUC for once-daily tacrolimus in the expressor group (Figure 4).

\section{Safety}

Adverse events (AEs) are summarized in Table 4. A total 71 AEs occurred in 35 out of 60 (58.3\%) patients included in the FAS. The distributions of AEs in the expressor and non-expressor groups did not differ significantly $(\mathrm{P}=0.796)$. Most $\mathrm{AEs}, 82.9 \%$ in the expressor group and $63.3 \%$ in the non-expressor group, were mild. However, the incidence of moderate severity AEs in the expressor group was lower than in the non-expressor group (14.6\% vs. $33.3 \%$ ). In the expressor group, $24.4 \%$ of the AEs had a probable or possible relationship to the study drug, and three patients experienced AEs that led to drug discontinuation. No AEs related to the study drug or requiring its discontinuation were recorded in the non-expressor group. While the incidence of AEs in the investigations and gastrointestinal systems was higher in the expressor group than in the non-expressor group, the study groups did not differ significantly in the incidence of AEs involving the other organ systems (Figure 5). Serious AEs involving the nervous system $(\mathrm{n}=1)$, investigations $(\mathrm{n}=2)$, and gastrointestinal system $(\mathrm{n}=3)$ occurred solely in the expressor group. Additionally, severe infectious complications were not developed in the patients.

\section{DISCUSSION}

The safety and efficacy of twice-daily and once-daily tacrolimus in liver transplant recipients were shown to be similar. ${ }^{3,10,14-16}$ Several previous studies demonstrated that liver dysfunction is the most common adverse 
event after converting from twice-daily to once-daily tacrolimus. ${ }^{11}$ In our present study, the incidence of liver dysfunction in stable liver transplant recipients converted to once-daily extended-release tacrolimus was higher in the CYP3A5 expressor group than in the non-expressor group. However, the between group difference was not statistically significant. None of the study patients experienced acute rejection or graft failure, and no mortality was observed in our series. In previous studies, CYP3A5expression was shown to be associated with a significant increase in the risk of biopsy-proven acute rejection and tacrolimus-induced nephrotoxicity at three months post-transplant, ${ }^{17}$ and with a decrease in eGFR at three months after renal transplantation, ${ }^{18}$ However, our present study did not demonstrate a significant difference in renal function in $C Y P 3 A 5$ expressors and non-expressors.

After 1:1 conversion from twice-daily to once-daily extended-release tacrolimus, the trough level of the drug in stable adult liver transplant recipients decreased by nearly $25 \%$. Up to $25 \%$ of the patients required a tacrolimus dose increase after the conversion. These findings are consistent with the results of other oncedaily tacrolimus conversion studies in LT patients, ${ }^{9-11,16}$ A 15-20\% increase in the daily dose of tacrolimus has been suggested in order to achieve the same target trough level of the drug following the conversion. ${ }^{19}$

CYP3A5 expression was shown to influence tacrolimus exposure, with the exposure in CYP3A5 expressors receiving either twice-daily tacrolimus or once-daily tacrolimus being lower than in the non-expressors. ${ }^{6-8,14}$ CYP3A 5 expressors carry the ${ }^{*} 1$ variant which encodes the functional enzyme responsible for the metabolism of tacrolimus; therefore, they may require a higher dose of tacrolimus than the non-expressors to achieve the target trough level of the drug. ${ }^{3-5}$ Our present study demonstrated that CYP3A5 polymorphism influenced both tacrolimus dose and trough level of the drug; the median dose of tacrolimus both before and after switching to the once-daily extended-release formulation was significantly higher in the expressor group than in the non-expressors. This observation is also consistent with the results of previous studies in which CYP3A5 expressors, both adult and pediatric patients, required higher doses of tacrolimus due to higher oral clearance of the drug. ${ }^{20,21}$ Our present study showed that the decrease in the trough level of tacrolimus was greater in the expressor group than in the non-expressors, also after adjustment for patient weight and tacrolimus dose. CYP3A5 genotype is known to play a role in determining the effect of interacting drugs, such as fluconazole, on tacrolimus pharmacokinetics. ${ }^{5}$ However, it needs to be stressed that our study did not analyze the issue of drug interaction, and all patients who received agents that might potentially interact with tacrolimus were excluded from the analysis.

While we did not find a significant difference in the incidence of AEs in the expressors and non-expressors, the frequency of drug-related AEs was higher in the former group. This was likely associated with a higher dose of tacrolimus received by the expressors. In view of this observation, a higher dose of tacrolimus might be a confounder in previous studies analyzing the link between $C Y P 3 A 5$ genotype and nephrotoxicity risk, especially given that our present study did not demonstrate a significant CYP3A5 genotype-related difference in the occurrence of renal dysfunction. In a recent study, Korean adult liver transplant recipients with CYP3A5 expression presented with low peripheral blood CD4+ adenosine triphosphate (ATP) immune response activity despite maintaining a constant concentration of tacrolimus, and suffered from infectious complications. ${ }^{22}$ In contrast, the incidence of infectious complications in our present study was lower in the expressor group than in the non-expressor group ( $7.1 \%$ vs. $18.3 \%)$, and no severe infections were recorded among the expressors.

Correlation between Cmin of tacrolimus and the effects of the drug is known to be stronger than the doseeffect correlation ${ }^{23}$. Because of a strong correlation between Cmin and systemic exposure (AUC), the dose of tacrolimus can be tailored using the Cmin level as a surrogate marker of exposure. ${ }^{3}$ Monitoring of Cmin is mandatory to minimize the risk of rejection (Cmin below the target range), as well as to reduce the risk of nephrotoxicity, and, to a lesser extent, neurotoxicity (Cmin above the target range). ${ }^{3,14}$ In present study, the AUC for twice-daily tacrolimus did not differ significantly from the AUC for once-daily tacrolimus, despite a lower Cmin for the latter. This implies that the same target trough level of tacrolimus cannot be used to predict the efficacy of the drug (AUC) after the conversion, especially among the expressors. In the non-expressor group, Cmin correlated significantly with AUC, whereas a significant correlation between 
Cmax and AUC was observed in the expressor group. Genetic polymorphisms are known to influence drug metabolism and have been implicated as a cause of individual variability in drug pharmacokinetics. A difference in $C Y P 3 A 5$ protein expression level in the small intestine, and replacement of croscarmellose with ethylcellulose may influence the oral bioavailability of tacrolimus in both initial exposure and steady state. Therefore, the diffusion rate of tacrolimus after administration of its once-daily formulation leads to a prolonged release of the drug. In our present study, the absorption of once-daily tacrolimus in CYP3A5nonexpressors was slower compared with the twice-daily formulation.

In a previous study, conversion from twice-daily to once-daily tacrolimus was associated with a $21 \%$ decrease in the median drug exposure in CYP3A5 expressors. Based on that observation, approximately a 1.25fold increase in total daily tacrolimus dose was recommended in CYP3A5 expressors after switching to the once-daily formulation, to maintain the same level of tacrolimus exposure. ${ }^{24}$ However, the results of our present study imply that the dose adjustment might not be necessary since, in the expressor group, the AUC after conversion to the once-daily extended-release tacrolimus did not differ significantly from that after the conversion, and unlike in the non-expressors, the AUC correlated with Cmax, rather than with Cmin.

This study has several limitations. First, the sample size was relatively small, and the follow-up period was quite short. Hence, further large-scale studies are needed to determine whether the events occurring during the post-transplant period might have an adverse effect on the long-term outcome in liver transplant recipients. Second, although we compared the incidence of liver dysfunction in the expressor and nonexpressor groups, reducing the drug concentration in this study increased the tacrolimus dose given by the physician to maintain an adequate trough level, which limits the analysis. Therefore, it was difficult to determine the true incidence of liver dysfunction. Third, our study was conducted with Koreans. Therefore, our results cannot be generalized to patients in Western countries. Fourth, the pharmacokinetic analysis included only ten patients, and previous studies documented a considerable intra- and interpatient variability in the pharmacokinetics of tacrolimus delivered in either twice-daily or once-daily formulations. ${ }^{19,25,26}$ Hence, the effects of CYP3A5 gene polymorphism on tacrolimus pharmacokinetics need to be verified in a larger group of liver transplant recipients. Fifth, we did not know the CYP3A5 gene status of liver donors. Unlike in other organ transplantations, the liver transplant does not need to share the genetic background of the recipient. As the activity of most drug-metabolizing enzymes is very elevated in the liver, polymorphism of the CYP3A5 gene, whether in the recipient or the donor, would likely contribute to individual variance in drug pharmacokinetics.

In conclusion, the results of the present study suggest that determination of the CYP3A5 genotype in liver transplant recipients might be helpful in both prediction of tacrolimus pharmacokinetics after conversion to once-daily extended-release tacrolimus formulation. After the conversion, CYP3A5 expressors showed a more evident decrease in the trough level of tacrolimus (Cmin) than the non-expressors. However, the pharmacokinetic analysis did not show a significant difference in the AUC before and after the conversion. The AUC in CYP3A5 expressors switched to once-daily tacrolimus correlated with Cmax, rather than with Cmin. This implies that CYP3A5 expression might have a greater influence on the pharmacokinetics of once-daily tacrolimus than the twice-daily tacrolimus formulation.

The clinical relevance of the findings presented above needs to be verified in further large-scale studies analyzing various pharmacogenetic strategies for tacrolimus dosing and the effect of $C Y P 3 A 5$ genetic polymorphism on long-term outcomes in liver transplant recipients.

\section{Financial support provided by Astellas Pharma Korea, Inc.}

The funders had no role in the study design, data collection, analysis, decision to publish, or manuscript preparation.

\section{Potential conflict of interest}

There are no competing interests to declare.

\section{Data availability statement}


The data that support the findings of this study are available from the corresponding author upon request.

\section{ORCID}

Jong Man Kim: 0000-0002-1903-8354

Je Ho Ryu: 0000-0002-9377-3074

Kwang-Woong Lee: 0000-0001-6412-1926

Kwangho Yang: 0000-0003-1489-6787

\section{REFERENCES}

1. Group USMFLS. A comparison of tacrolimus (FK 506) and cyclosporine for immunosuppression in liver transplantation. N Engl J Med . 1994;331(17):1110-1115.

2. Kuypers DRJ. Intra-Patient Variability of tacrolimus exposure in solid organ transplantation: a novel marker for clinical outcome. Clin Pharmacol Ther . 2019.

3. Brunet M, van Gelder T, Asberg A, et al. Therapeutic Drug Monitoring of Tacrolimus-Personalized Therapy: Second Consensus Report. Ther Drug Monit . 2019;41(3):261-307.

4. Schutte-Nutgen K, Tholking G, Suwelack B, Reuter S. Tacrolimus - Pharmacokinetic Considerations for Clinicians. Curr Drug Metab . 2018;19(4):342-350.

5. Hendijani F, Azarpira N, Kaviani M. Effect of CYP3A $5 * 1$ expression on tacrolimus required dose after liver transplantation: A systematic review and meta-analysis. Clin Transplant . 2018;32(8):e13306.

6. Loh PT, Lou HX, Zhao Y, Chin YM, Vathsala A. Significant impact of gene polymorphisms on tacrolimus but not cyclosporine dosing in Asian renal transplant recipients. Transplant Proc . 2008;40(5):1690-1695.

7. Ji E, Kim MG, Oh JM. CYP3A5 genotype-based model to predict tacrolimus dosage in the early postoperative period after living donor liver transplantation. Ther Clin Risk Manag . 2018;14:2119-2126.

8. Chen L, Prasad GVR. CYP3A5 polymorphisms in renal transplant recipients: influence on tacrolimus treatment. Pharmgenomics Pers Med . 2018;11:23-33.

9. Kim SH, Lee SD, Kim YK, Park SJ. Conversion of twice-daily to once-daily tacrolimus is safe in stable adult living donor liver transplant recipients. Hepatobiliary Pancreat Dis Int . 2015;14(4):374-379.

10. Kim JM, Kwon CH, Joh JW, et al. Conversion of once-daily extended-release tacrolimus is safe in stable liver transplant recipients: A randomized prospective study. Liver Transpl . 2016;22(2):209-216.

11. Suh SW, Lee KW, Jeong J, Kim H, Yi NJ, Suh KS. Risk Factors for the Adverse Events after Conversion from Twice-Daily to Once-Daily Tacrolimus in Stable Liver Transplantation Patients. J Korean Med Sci . 2016;31(11):1711-1716.

12. Demetris AJ, Bellamy C, Hubscher SG, et al. 2016 Comprehensive Update of the Banff Working Group on Liver Allograft Pathology: Introduction of Antibody-Mediated Rejection. Am J Transplant . 2016;16(10):2816-2835.

13. Demetris A, Adams D, Bellamy C, et al. Update of the International Banff Schema for Liver Allograft Rejection: working recommendations for the histopathologic staging and reporting of chronic rejection. An International Panel. Hepatology . 2000;31(3):792-799.

14. Iwasaki M, Yano I, Fukatsu S, et al. Pharmacokinetics and Pharmacodynamics of Once-Daily Tacrolimus Compared With Twice-Daily Tacrolimus in the Early Stage After Living Donor Liver Transplantation. Ther Drug Monit . 2018;40(6):675-681. 
15. Fischer L, Trunecka P, Gridelli B, et al. Pharmacokinetics for once-daily versus twice-daily tacrolimus formulations in de novo liver transplantation: a randomized, open-label trial. Liver Transpl . 2011;17(2):167177.

16. Beckebaum S, Iacob S, Sweid D, et al. Efficacy, safety, and immunosuppressant adherence in stable liver transplant patients converted from a twice-daily tacrolimus-based regimen to once-daily tacrolimus extended-release formulation. Transpl Int . 2011;24(7):666-675.

17. Kuypers DR, Naesens M, de Jonge H, Lerut E, Verbeke K, Vanrenterghem Y. Tacrolimus dose requirements and CYP3A5 genotype and the development of calcineurin inhibitor-associated nephrotoxicity in renal allograft recipients. Ther Drug Monit . 2010;32(4):394-404.

18. Genvigir FD, Salgado PC, Felipe CR, et al. Influence of the CYP3A4/5 genetic score and ABCB1 polymorphisms on tacrolimus exposure and renal function in Brazilian kidney transplant patients. Pharmacogenet Genomics . 2016;26(10):462-472.

19. Tremblay S, Nigro V, Weinberg J, Woodle ES, Alloway RR. A Steady-State Head-to-Head Pharmacokinetic Comparison of All FK-506 (Tacrolimus) Formulations (ASTCOFF): An Open-Label, Prospective, Randomized, Two-Arm, Three-Period Crossover Study. Am J Transplant . 2017;17(2):432-442.

20. Min SI, Kim SY, Ahn SH, et al. CYP3A $5 * 1$ allele: impacts on early acute rejection and graft function in tacrolimus-based renal transplant recipients. Transplantation . 2010;90(12):1394-1400.

21. Barry A, Levine M. A systematic review of the effect of CYP3A5 genotype on the apparent oral clearance of tacrolimus in renal transplant recipients. Ther Drug Monit . 2010;32(6):708-714.

22. Mizuno S, Hamada T, Nakatani K, et al. Monitoring peripheral blood CD4+ adenosine triphosphate activity after living donor liver transplantation: impact of combination assays of immune function and CYP3A5 genotype. J Hepatobiliary Pancreat Sci . 2011;18(2):226-232; discussion 232-224.

23. Woillard JB, de Winter BC, Kamar N, Marquet P, Rostaing L, Rousseau A. Population pharmacokinetic model and Bayesian estimator for two tacrolimus formulations-twice daily Prograf and once daily Advagraf.Br J Clin Pharmacol . 2011;71(3):391-402.

24. Andrews LM, Li Y, De Winter BCM, et al. Pharmacokinetic considerations related to therapeutic drug monitoring of tacrolimus in kidney transplant patients. Expert Opin Drug Metab Toxicol . 2017;13(12):12251236 .

25. Caillard S, Moulin B, Buron F, et al. Advagraf $((\mathrm{R}))$, a once-daily prolonged release tacrolimus formulation, in kidney transplantation: literature review and guidelines from a panel of experts. Transpl Int . 2016;29(8):860-869.

26. Shuker N, Cadogan M, van Gelder T, et al. Conversion from twice-daily to once-daily tacrolimus does not reduce intrapatient variability in tacrolimus exposure. Ther Drug Monit . 2015;37(2):262-269.

Table 1. Baseline characteristics of the study patients stratified according to CYP3A5 expression.

\begin{tabular}{llll}
\hline & Expressor group $(\mathrm{n}=28)$ & $\begin{array}{l}\text { Non-expressor group }(\mathrm{n} \\
=32)\end{array}$ & P-value \\
\hline $\begin{array}{l}\text { Donor } \\
\text { Sex (Male) }\end{array}$ & $12(42.9 \%)$ & $24(77.4 \%)$ & 0.008 \\
Age (years) & $34(18-68)$ & $33(20-78)$ & 0.641 \\
Recipient & & & \\
Sex (Male) & $18(64.3 \%)$ & $25(78.1 \%)$ & 0.264 \\
Age (years) & $57(35-74)$ & $54(43-71)$ & 0.870
\end{tabular}




\begin{tabular}{|c|c|c|c|}
\hline & Expressor group $(\mathrm{n}=28)$ & $\begin{array}{l}\text { Non-expressor group ( } \mathrm{n} \\
=32 \text { ) }\end{array}$ & P-value \\
\hline $\begin{array}{l}\text { Indications for LT } \\
\text { Alcoholic HBV HCV } \\
\text { HCC AIH Biliary } \\
\text { cirrhosis Budd-Chiari } \\
\text { syndrome NBNC PBC } \\
\text { PSC Wilson's disease }\end{array}$ & 45212100020002 & 93312111101110 & 0.396 \\
\hline Retransplantation & $0(0 \%)$ & $1(3.1 \%)$ & 1.000 \\
\hline Type of LT (LDLT) & $20(71.4 \%)$ & $20(62.5 \%)$ & 0.585 \\
\hline MELD & $15(6-40)$ & $14(6-40)$ & 0.406 \\
\hline $\begin{array}{l}\text { Time from LT to study } \\
\text { enroll (mo.) }\end{array}$ & $16.7(6.9-35.5)$ & $16.6(6.2-43.5)$ & 0.711 \\
\hline
\end{tabular}

* LT, liver transplantation; HBV, hepatitis B virus; $\mathrm{HCV}$, hepatitis $\mathrm{C}$ virus; $\mathrm{HCC}$, hepatocellular carcinoma; $\mathrm{AIH}$, autoimmune hepatitis; NBNC, non-B non-C; PBC, primary biliary cirrhosis; PSC, primary sclerosing cholangitis; LDLT, living donor liver transplantation; MELD, model for end-stage liver disease

Table 2. Tacrolimus exposure parameters in CYP3A5 expressors and non-expressors before and after the conversion from twice-daily to once-daily extended-release formulation.

\begin{tabular}{|c|c|c|c|}
\hline & Before & After & $\mathrm{P}$-value \\
\hline \multicolumn{4}{|l|}{ Expressor group $(\mathrm{n}=28)$} \\
\hline Tacrolimus trough level (Cmin) $(\mathrm{ng} / \mathrm{mL})$ & $5.0(2.6-7.9)$ & $3.8(0.6-5.0)$ & $<0.001$ \\
\hline Dose-adjusted Cmin $(\mathrm{ng} / \mathrm{mL} / \mathrm{mg})$ & $1.4(0.7-3.2)$ & $0.8(0.4-2.8)$ & $<0.001$ \\
\hline Dose $/ \mathrm{kg}$-adjusted Cmin (ng/mL/mg/kg) & $0.025(0.008-0.067)$ & $0.015(0.005-0.048)$ & $<0.001$ \\
\hline \multicolumn{4}{|l|}{ Non-expressor group $(\mathrm{n}=32)$} \\
\hline Tacrolimus trough level (Cmin) $(\mathrm{ng} / \mathrm{mL})$ & $4.7(2.7-7.8)$ & $3.9(1.8-7.1)$ & $<0.001$ \\
\hline Dose-adjusted Cmin (ng/mL/mg) & $2.3(1.0-6.2)$ & $1.7(0.7-4.7)$ & $<0.001$ \\
\hline Dose $/ \mathrm{kg}$-adjusted Cmin $(\mathrm{ng} / \mathrm{mL} / \mathrm{mg} / \mathrm{kg})$ & $0.038(0.016-0.115)$ & $0.029(0.011-0.078)$ & $<0.001$ \\
\hline
\end{tabular}

Table 3. Pharmacokinetic parameters of tacrolimus stratified according to CYP3A5 expression.

\begin{tabular}{|c|c|c|c|}
\hline & Expressor group $(\mathrm{n}=5)$ & $\begin{array}{l}\text { Non-expressor group (n } \\
=5)\end{array}$ & $\mathrm{P}$-value \\
\hline Dose (mg) & $4(3-5)$ & $2(1-3)$ & 0.016 \\
\hline Weight (kg) & $54(51.3-78.0)$ & $65(43-82)$ & 0.690 \\
\hline Dose/Weight (mg/kg) & $0.077(0.045-0.097)$ & $0.037(0.016-0.047)$ & 0.032 \\
\hline $\begin{array}{l}\text { AUC in Tac BID } \\
(\mathrm{ng} \cdot \mathrm{hr} / \mathrm{mL})\end{array}$ & $126.3(98.2-146.9)$ & $107.0(102.4-126.7)$ & 0.310 \\
\hline $\begin{array}{l}\text { AUC in Tac QD } \\
(\mathrm{ng} \cdot \mathrm{hr} / \mathrm{mL})\end{array}$ & $112.5(81.0-168.6)$ & $96.1(68.5-131.7)$ & 0.310 \\
\hline $\begin{array}{l}\text { Cmin in Tac BID } \\
(\mathrm{mg} / \mathrm{mL})\end{array}$ & $3.77(3.05-4.20)$ & $3.07(2.76-4.47)$ & 0.421 \\
\hline $\begin{array}{l}\text { Cmin in Tac QD } \\
(\mathrm{mg} / \mathrm{mL})\end{array}$ & $3.04(1.85-4.21)$ & $2.59(1.81-4.47)$ & 0.690 \\
\hline $\begin{array}{l}\text { Cmax in Tac BID } \\
(\mathrm{mg} / \mathrm{mL})\end{array}$ & $8.20(4.94-11.54)$ & $9.93(5.08-10.48)$ & 0.690 \\
\hline
\end{tabular}




\begin{tabular}{|c|c|c|c|}
\hline & Expressor group $(\mathrm{n}=5)$ & $\begin{array}{l}\text { Non-expressor group (n } \\
=5)\end{array}$ & P-value \\
\hline $\begin{array}{l}\text { Cmax in Tac QD } \\
(\mathrm{mg} / \mathrm{mL})\end{array}$ & $9.52(6.81-11.90)$ & $7.85(3.54-10.86)$ & 0.151 \\
\hline $\begin{array}{l}\text { Dose-adjusted Cmin in } \\
\text { Tac BID (ng/mL/mg) }\end{array}$ & $0.918(0.754-1.050)$ & $1.535(0920-3.400)$ & 0.056 \\
\hline $\begin{array}{l}\text { Dose-adjusted Cmin in } \\
\text { Tac QD }(\mathrm{ng} / \mathrm{mL} / \mathrm{mg})\end{array}$ & $0.680(0.608-1.053)$ & $1.295(0.603-2.235)$ & 0.151 \\
\hline $\begin{array}{l}\text { Dose-adjusted Cmax in } \\
\text { Tac BID (ng/mL/mg) }\end{array}$ & $1.65(1.58-2.40)$ & $3.79(3.31-5.16)$ & 0.008 \\
\hline $\begin{array}{l}\text { Dose-adjusted Cmax in } \\
\text { Tac QD }(\mathrm{ng} / \mathrm{mL} / \mathrm{mg})\end{array}$ & $2.73(1.36-3.10)$ & $3.54(1.85-5.43)$ & 0.310 \\
\hline $\begin{array}{l}\text { Dose-adjusted AUC in } \\
\text { Tac BID } \\
(\mathrm{ng} \cdot \mathrm{hr} / \mathrm{mL} / \mathrm{mg})\end{array}$ & $29.4(25.3-33.0)$ & $53.5(35.2-102.4)$ & 0.008 \\
\hline $\begin{array}{l}\text { Dose-adjusted AUC in } \\
\text { Tac QD } \\
\text { (ng.hr/mL/mg) }\end{array}$ & $27.0(21.0-42.2)$ & $48.0(24.2-68.5)$ & 0.095 \\
\hline $\begin{array}{l}\text { Dose } / \mathrm{kg} \text {-adjusted } \\
\text { Cmin Tac BID } \\
\text { (ng/mL/mg/kg) }\end{array}$ & $0.015(0.013-0.018)$ & $0.021(0.012-0.053)$ & 0.548 \\
\hline $\begin{array}{l}\text { Dose/kg-adjusted } \\
\text { Cmin Tac QD } \\
\text { (ng/mL/mg/kg) }\end{array}$ & $0.013(0.009-0.014)$ & $0.017(0.009-0.052)$ & 0.222 \\
\hline $\begin{array}{l}\text { Dose/kg-adjusted } \\
\text { Cmax Tac BID } \\
(\mathrm{ng} / \mathrm{mL} / \mathrm{mg} / \mathrm{kg})\end{array}$ & $0.032(0.020-0.046)$ & $0.069(0.040-0.088)$ & 0.032 \\
\hline $\begin{array}{l}\text { Dose } / \mathrm{kg} \text {-adjusted } \\
\text { Cmax Tac QD } \\
\text { (ng/mL/mg/kg) }\end{array}$ & $0.038(0.025-0.052)$ & $0.053(0.029-0.126)$ & 0.421 \\
\hline $\begin{array}{l}\text { Dose/kg-adjusted AUC } \\
\text { Tac BID } \\
\text { (ng. } \mathrm{hr} / \mathrm{mL} / \mathrm{mg} / \mathrm{kg} \text { ) }\end{array}$ & $0.492(0.423-0.553)$ & $0.716(0.455-1.600)$ & 0.151 \\
\hline $\begin{array}{l}\text { Dose/kg-adjusted AUC } \\
\text { Tac QD } \\
\text { (ng.hr/mL/mg/kg) }\end{array}$ & $0.458(0.390-0.541)$ & $0.643(0.379-1.532)$ & 0.421 \\
\hline
\end{tabular}

* AUC, area under the curve; hr, hour; Tac, tacrolimus; BID, bid in die (twice daily); QD, quaque die (once daily)

\section{FIGURE LEGENDS}

Figure 1. Study population. FAS, full analysis set; PP, per-protocol set.

Figure 2. Laboratory parameters of CYP3A5 expressors and non-expressors determined during regular control visits in per-protocol set (medians with 95\% confidence interval). (A) Aspartate aminotransferase (AST), (B) Alanine aminotransferase (ALT), (C) total bilirubin, (D) Alkaline phosphatase (ALP).

Figure 3. Tacrolimus exposure in CYP3A5 expressors and non-expressors determined during regular control visits in per-protocol set (medians with 95\% confidence interval). (A) trough level of tacrolimus, (B) daily tacrolimus dose, (C) tacrolimus dose/weight, (D) dose-adjusted C0, (E) dose/kg-adjusted C0. 
Figure 4. (A) 24-hour arithmetic mean whole blood tacrolimus concentrations (ng/mL) and standard deviation for the twice-daily and once-daily formulations; (B) Correlation between the area under the curve (AUC) and Cmin or Cmax in the expressor group after conversion to once-daily extended-release tacrolimus; (C) Correlation between the area under the curve (AUC) and Cmin or Cmax in the non-expressor group after conversion to once-daily extended-release tacrolimus.

Figure 5. Adverse event profiles in the expressors and non-expressors (Full Analysis Set) after conversion from twice-daily to once-daily extended-release tacrolimus.

Table 4. Adverse events stratified according to CYP3A5 expression

\begin{tabular}{llll}
\hline & Expressor $(\mathrm{n}=28)$ & Non-expressor $(\mathrm{n}=32)$ & P-value \\
\hline $\begin{array}{l}\text { Patients with adverse } \\
\text { events }\end{array}$ & $17(60.7 \%)$ & $18(56.3 \%)$ & 0.796 \\
$\begin{array}{l}\text { Adverse events } \\
\text { Severity Mild Moderate }\end{array}$ & $41(82.9 \%) 6(14.6 \%) 1$ & $19(63.3 \%) 10(33.3 \%) 1$ & 0.070 \\
$\begin{array}{l}\text { Severe } \\
\text { Related to the study drug }\end{array}$ & $(2.4 \%)$ & $(3.3 \%)$ & \\
$\begin{array}{l}\text { Certain Probable/likely } \\
\text { Possible Unlikely }\end{array}$ & $(12.2 \%) 30(73.2 \%) 1$ & $(100 \%) 0(0 \%) 0(0 \%)$ & \\
$\begin{array}{l}\text { Conditional, unclassified } \\
\begin{array}{l}\text { Unassessable/unclassifiable } \\
\text { Subjects with adverse }\end{array}\end{array}$ & $3(10.7 \%)$ & & \\
$\begin{array}{l}\text { events leading to drug } \\
\text { discontinuation }\end{array}$ & & $0(0 \%)$ & 0.023 \\
\hline
\end{tabular}

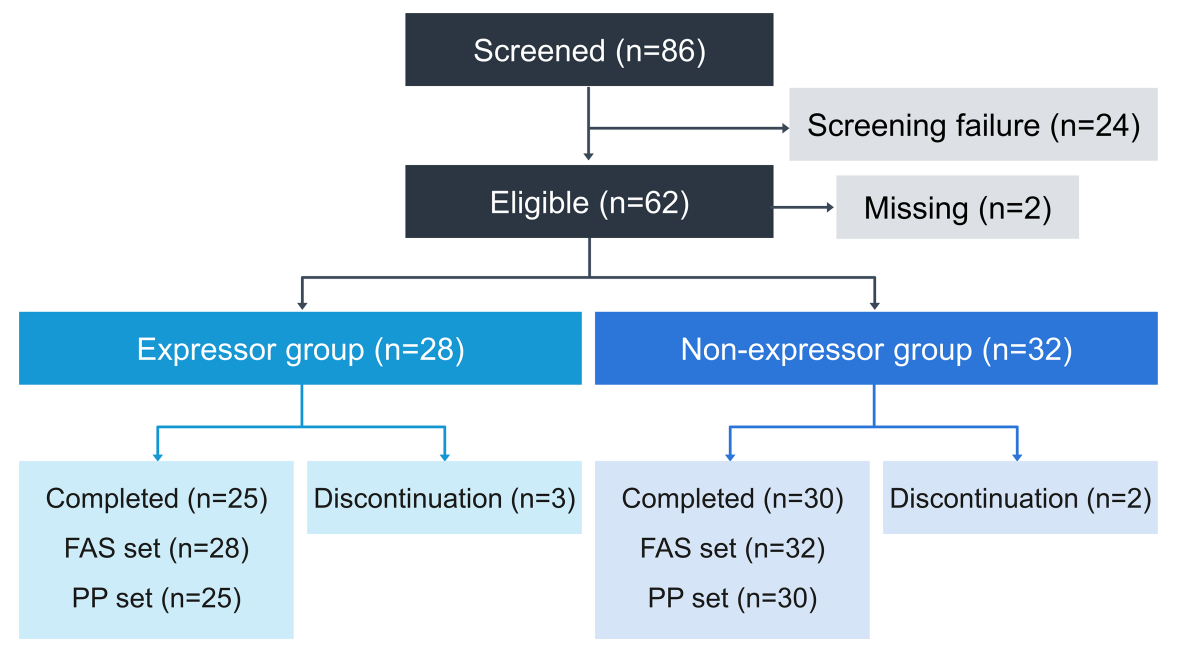



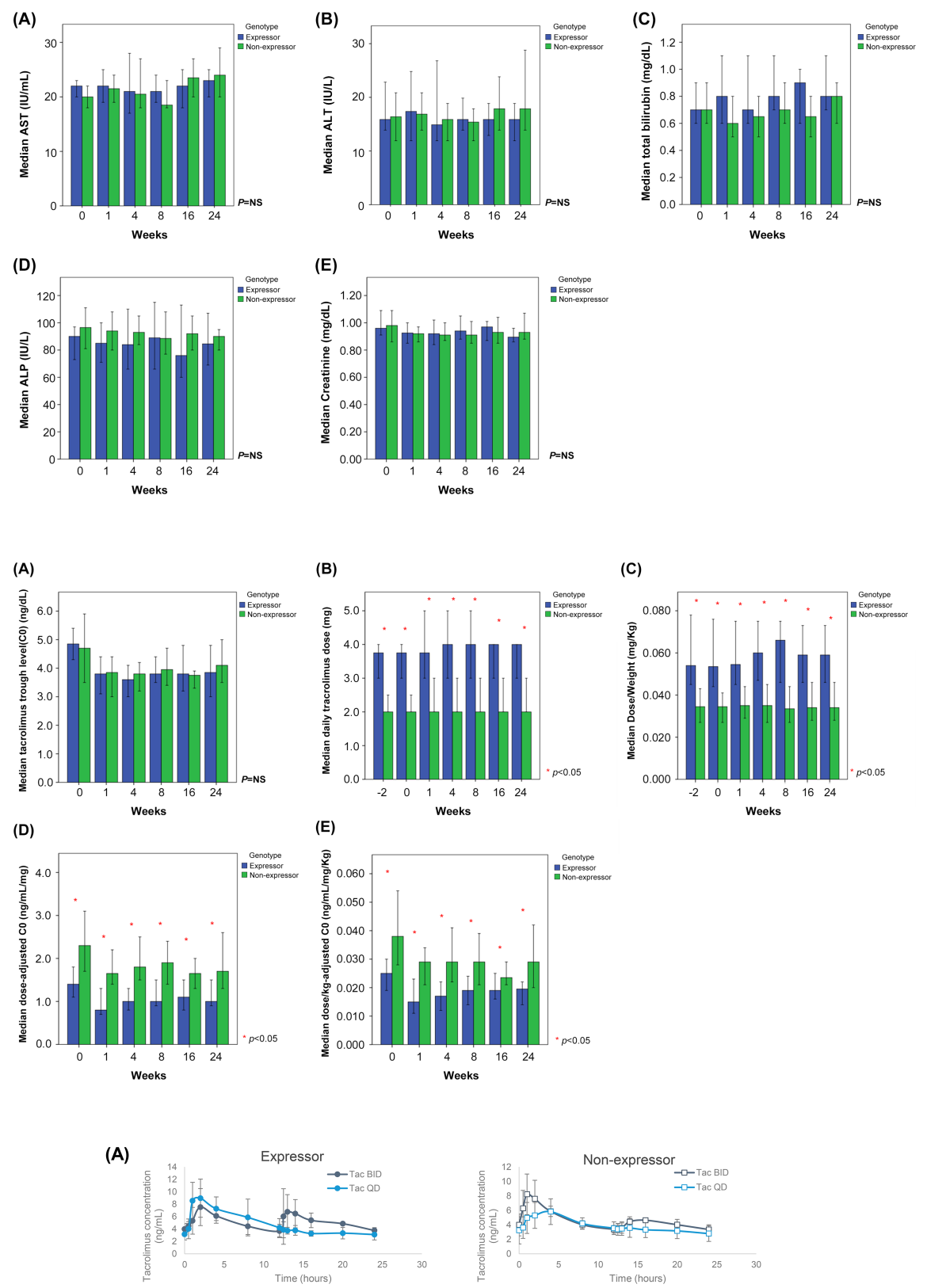

(B)

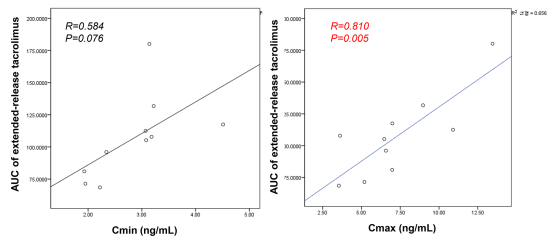

(C)

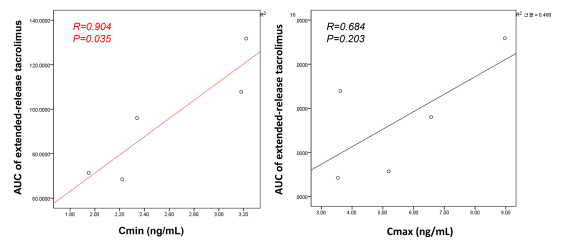




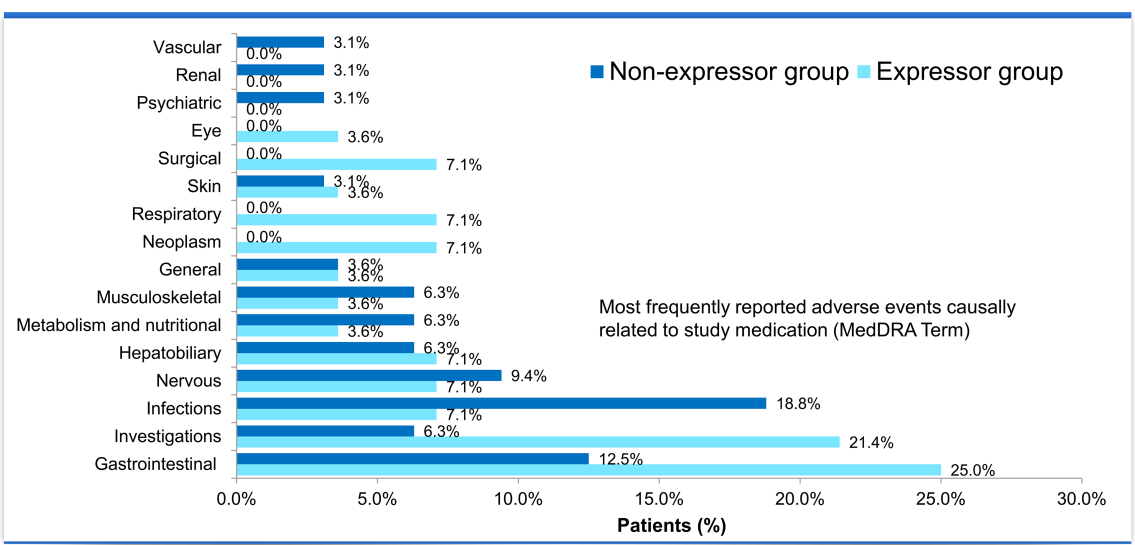

